\title{
LA EVALUACIÓN FORMATIVA Y COOPERATIVA EN EDUCACIÓN FÍSICA
}

\author{
Formative assessment and Cooperative learning in Physical Education \\ Avaliação formativa e cooperativa em Educação Física
}

\section{Antonio Fraile Aranda}

Universidad de Valladolid, España. Fono: +34 983343957. Correo electrónico: afraile@mpc.uva.es

\section{Resumen}

En este trabajo se recoge una experiencia de evaluación formativa, que se viene realizando en la asignatura de Expresión Corporal, dentro del programa de formación inicial del profesorado de Educación Física en la Facultad de Educación en la Universidad de Valladolid. Esta asignatura se desarrolla a partir de un modelo de aprendizaje colaborativo, en donde los estudiantes asumen funciones que, habitualmente, son responsabilidad del profesorado. En este caso, se presentan y analizan las estrategias y procedimientos que aplican los estudiantes en un proceso de coevalución. Los estudiantes señalan en su cuaderno de campo las ventajas e inconvenientes que supone el uso de esta metodología. Los resultados más relevantes señalan la importancia que tiene para el alumnado esta experiencia, como un proceso formativo que exige del trabajo en equipo y de forma colaborativa. Sin embargo, entre los inconvenientes destacan la exigencia de un trabajo continuado y a diario y, por ello, la asistencia a clase debe ser obligatoria y activa.

Palabras claves: evaluación formativa, aprendizaje cooperativo, Educación Física, formación del profesorado.

\begin{abstract}
In this work an experience of formative assessment that has been done on the subject of body language, within the program of initial teacher of Physical Education in the Faculty of Education at the University of Valladolid is collected. This course is developed from a model of collaborative learning, where students assume functions that are normally the responsibility of the teacher. In this case, they present and analyzes the strategies and procedures applied by the students in a process of coevalución. Students say in his field notebook the advantages and disadvantages to using this methodology. The most relevant results indicate the importance of this experience for the students as a
\end{abstract}


learning process that requires the work in a collaborative team. However, among the disadvantages include the requirement of a daily and continuous work and, therefore, the attendance should be mandatory and active.

Keywords: Formative assessment, Cooperative learning, Physical Education, Teacher training.

\section{Resumo}

Neste trabalho uma experiência de avaliação formativa que tem sido feito sobre o tema da expressão corporal, dentro do programa de formação inicial de professores de Educação Física na Faculdade de Educação da Universidade de Valladolid. Este curso usos um modelo de aprendizagem colaborativa, onde os alunos assumem funções que normalmente são da responsabilidade dos professores. Neste caso, apresentar e analisar as estratégias e procedimentos de coevalución aplicados pelos alunos. Os estudantes dizem em seu caderno de campo as vantagens e desvantagens de se utilizar esta metodologia. Os resultados mais relevantes indicam a importância dessa experiência para os alunos como um aprendizagem que exige trabalho em equipe e colaboração. No entanto, entre as desvantagens incluem a exigência de um trabalho diário e contínuo e, portanto, a presença deve ser obrigatória e ativa.

Palavras-chave: Avaliação formativa, A aprendizagem cooperativa, Educação Física, Formação de professores.

\section{Introducción}

Con la declaración de La Sorbona en 1998 aparece, por primera vez, la voluntad de potenciar una Europa del conocimiento, con el objetivo de que la educación superior posibilite que los estudiantes, como futuros ciudadanos, puedan alcanzar una mejor calidad de vida, como valor prioritario de la sociedad del bienestar (Fraile, 2009a).

Esa construcción del conocimiento pasa por un proceso complejo, a partir de tres elementos: el estudiante que aprende a través de la actividad mental, el contenido de enseñanza y aprendizaje, y unos docentes que ayudan al estudiante, en dicho proceso, aportando un mayor significado a lo que aprenden (Morin, 2000). De modo que el docente, los estudiantes y los contenidos configuran un triángulo interactivo, donde el 
nuevo aprendizaje se conecta con el antiguo, a través de diversos procedimientos de enseñanza-aprendizaje.

De acuerdo con Dewey, el objetivo de la educación debe ser educar para la libertad y la democracia, superando aquellos modelos tradicionales, dependiente de las instituciones dominantes que solamente están preocupadas por la subordinación y la marginalización (Chomsky, 2001). Pero esas prácticas democráticas deben estar reflejadas en todos aquellos momentos y espacios de la actividad docente, poniendo énfasis en lo que aprende el estudiante e incorporando estrategias como: la negociación del currículo, el trabajo cooperativo entre profesor-estudiantes y la evaluación formativa. Desde unos principios humanistas que fomenten el respeto por la dignidad de los estudiantes, como proceso democrático (Marina, 1995). Así como desde políticas que exijan una alta cualificación del profesorado a través de enfoques multidisciplinares, que ayuden a conectar las necesidades de los educandos con entornos sociales en constante cambio.

Por tanto, las tareas de enseñar-aprender en el espacio educativo superior no se entienden sin procedimientos, como el trabajo cooperativo, que incidan en la mejora del pensamiento crítico. Siendo la comunicación y la interacción, con y entre los alumnos, una de las competencias claves de la actividad profesional. A su vez, el desarrollo científico y profesional de las nuevas enseñanzas requiere que estas nuevas competencias profesionales se alíen con la mejora social, utilizando nuevas estrategias docentes que permitan intervenir más eficientemente en el aula, ya que la función de la enseñanza ya no sólo debe representar la acción de transmitir un conocimiento adaptado a esas nuevas exigencias sociales, sino generar un tipo de relaciones democráticas con los estudiantes, como futuros ciudadanos de una aldea global.

\section{La evaluación formativa como base del aprendizaje}

Uno de los componente básicos de ese proceso educativo democratizador es la tarea de evaluar que, tradicionalmente, se ha venido identificando como una actividad finalista y medidora de los resultados de los estudiantes. Según recoge Álvarez Méndez (1999), el interés de algunos profesores por evaluar al alumnado con la máxima objetividad ha venido perjudicando la comunicación y el diálogo, impidiendo una participación más democrática en el aula. 
Como alternativa a ese proceso anclado en los modelos reproductores del conocimiento, debemos buscar un tipo de evaluación en la que el alumnado asuma un importante protagonismo, en donde se invite a los estudiantes a analizar y reflexionar sobre su aprendizaje (desde los conocimientos previos hasta el resultado final), ayudándoles a identificar sus carencias, a reconocer sus errores y ser conscientes de sus progresos. La evaluación, por tanto, debe representa una de las claves del aprendizaje activo y democrático, a través de la cual el alumnado alcance un mayor autoconocimiento personal.

El proceso evaluativo en la enseñanza en general y particularmente en la Educación Superior tradicionalmente ha venido siendo, con exclusividad, labor del profesorado; solamente, con las nuevas tendencias en evaluación se aprecia, cada vez más, la participación democrática del alumnado. Gracias a poder superar, como única vía, la evaluación sumativa. Es la evaluación formativa la herramienta que facilitará un aprendizaje más autónomo y continuado (Boud y Falchikov, 2007; Brown y Glasner, 2007 y Falchikov, 2005), y donde los estudiantes adquieren, cada vez, un mayor protagonismo. La utilización de nuevos procesos como la autoevaluación, la autocalificación, la evaluación compartida y calificación dialogada en la enseñanza universitaria en nuestro país, referidos en este caso a la formación inicial del profesorado de educación física (Fernández-Balboa, 2005; 2006; Fraile, 2006, 2009b; López et al, 2006; 2009), son unos buenos referentes de ese carácter formativo que debe adoptar la nueva forma de evaluar.

Esos nuevos proceso de evaluar deben estar apoyados en procedimientos, pruebas e instrumentos renovados que nos faciliten un mejor acceso al conocimiento del alumnado, entre ellos destacaremos los siguientes: el portafolios, la autoevaluación y la coevaluación entre iguales, los ensayos, los informes escritos, las memorias, las pruebas orales, los exámenes, los incidentes críticos y la observación de habilidades prácticas (Brown et al., 1995; Barberá, 1999; Bordás Alsina y Cabrera Rodríguez, 2001; Brown, 2003; MacDowell y Sambell, 2003; Fraile, 2004; López Pastor, 2009; Ibarra et al. 2012).

Una de las características de la evaluación formativa, diferenciadora de la evaluación tradicional, es la que aportan algunos autores, entre ellos Monereo (2009) y Cano (2005), que consideran que este nuevo proceso formativo representa la evaluación 
auténtica. En el estudio realizado por Tejedor (2002), los profesores definen la evaluación formativa a partir de los siguientes conceptos: es autocorrectora del aprendizaje del alumnado; desarrolla el pensamiento creador del alumnado; evalúa continuamente los conocimientos adquiridos y nos informa de inmediato de los resultados del alumnado.

La evaluación formativa y compartida posee numerosas ventajas de cara a mejorar la calidad de la enseñanza de la enseñanza superior, ya que incide de forma clara en la mejora de los procesos de aprendizaje del alumnado; favorece la motivación del alumnado hacía el aprendizaje; mejora la responsabilidad y autonomía del alumnado en los procesos de aprendizaje, con lo que adquiere un gran potencial de cara al desarrollo de estrategias de aprendizaje permanente e incrementa la calidad docente, el rendimiento académico del alumnado y los niveles de éxito escolar (Bordás y Cabrera, 2001). Por tanto, este tipo de evaluación se puede considerar como una de las formas más efectivas para promover la colaboración y cooperación entre los estudiantes (Prins et al., 2005), a través del diálogo, la interacción y la creación de significados comunes entre los miembros del grupo. Para Falchikov y Goldfinch (2000) la evaluación entre iguales se puede utilizar con éxito en cualquier disciplina, área y nivel; siendo diferente la presencia de las diversas estrategias docentes. Así, en trabajos de Dochy, et al., (1999); Falchikov (2001) y Brew (2003) se señala cómo es la participación y el grado de implicación de los estudiantes en dicho sistema coevaluativo.

Por último y a modo de conclusión, Bain (2006) destaca que sin una evaluación adecuada, ni los profesores ni los estudiantes pueden llegar a conocer y comprender el progreso que se viene produciendo en la manera de enseñar y en lo que ha sido objeto de aprendizaje. Es decir, la evaluación formativa debe proporcionar al profesor información que le permita intervenir para mejorar y reorientar su propio proceso de enseñanza, disponiendo de una mejor visión de las dificultades y progresos de los estudiantes. Mientras que los estudiantes pueden llegar a conocerse mejor como aprendices y pensadores, y no sólo estar preocupados por la adquisición de conocimientos. 


\section{El aprendizaje cooperativo como competencia}

Otra de las cuestiones claves para desarrollar en la enseñanza superior un proceso de enseñar-aprender democrático es el aprendizaje colaborativo. Johnson y Johnson (1975) consideran que los alumnos cuando aprenden juntos tienen más posibilidades de resolver los problemas, ya que pueden compartir significados que les ayuda a aprender unos de otros. No obstante, este exige que el docente adopte un estilo pedagógico más activo, como facilitador, a la hora de organizar y dinamizar sus clases.

La enseñanza cooperativa precisa que los estudiantes y profesores actuemos de forma conjunta, desde procedimientos que nos ayuden a compartir significados. Aunque, en muchos momentos, el profesor deba ir guiando las actividades que permitan a los alumnos descubrir los elementos del programa, ya que la actuación colaborativa requiere de la resolución de problemas en grupo, desde un consenso. Estudios sobre estos procedimientos cooperativos nos indican que entre los alumnos que aprenden en grupos, se reduce las conductas individuales y la intolerancia entre sus participantes (Johnston, 1997).

Del mismo modo, la mejora profesional de los docentes depende de su capacidad para desarrollar acciones colaborativas con otros profesores y con el propio alumnado, con objeto de intercambian conocimientos y experiencias (Stenhouse, 1987). Siendo labor del profesor diseñar instrumentos para reconocer: cómo los estudiantes se organizan en grupo, qué labores asumen cada uno de sus integrantes, qué obstáculos deben superar como colectivo, qué estrategias comparten durante su aprendizaje, etc.

Como parte de ese aprendizaje cooperativo, debemos invitar a los estudiantes, desde la primeras etapas educativas, a participar asumiendo algunas tareas, habitualmente reservadas para los docentes (Velázquez et al, 2014). Por ejemplo seleccionar: las finalidades de la materia, los contenidos de la enseñanza (que representarán los medios para lograr dichos fines), la metodología (procedimientos que incidan en el trabajo grupal), los criterios para su (auto)evaluación. Ese proceso de compartir y negociar las actividades de aprendizaje debe hacerse desde un principio de igualdad (Freire, 1970); así, el diálogo igualitario y tolerante entre profesor y alumnos se debe desarrollar superando las relaciones de poder del docente (Flecha, 1998). 
También Johnson et al., (1999), se refieren a la cooperación como una estrategia para que los estudiantes trabajen juntos y logren objetivos comunes. Y para ello, se deben cumplir las siguientes condiciones:

- El éxito individual depende de la actuación grupal y a la inversa.

- Cada miembro debe ser capaz de comunicar lo logros conseguidos por el grupo.

- Desarrollar dinámicas de ayuda, apoyo y refuerzo entre los miembros del equipo.

- Fomentar habilidades sociales que faciliten el trabajo cooperativo (resolución de conflictos, adoptar la perspectiva del otro, etc.).

- Valerse de una evaluación grupal que permita que los miembros de grupo puedan reflexionar colectivamente sobre el proceso desarrollado, tomando decisiones de forma conjunta.

A partir de la teoría de la comunicación de Habermas (1989), se destaca la actividad de comunicación e interacción con y entre los alumnos, ya que nos facilita poder construir y reestructurar nuestro conocimiento, como parte de un desarrollo cognitivo, social y emocional (componentes básicos para una educación integral). A través de ello, los estudiantes aprenden a: analizar, comparar, clasificar, interpretar, inferir, deducir, sintetizar, aplicar, valorar, etc., con medio para obtener un aprendizaje más comprensivo.

El incremento de los canales de información, en la sociedad del conocimiento, nos lleva ante la necesidad de trasladar al aula el dominio de esas competencias relacionadas con la comunicación verbal y no verbal, así como la escucha activa. Siendo necesario enseñar a cómo organizar esa información y a cómo presentarla en clase o a construirla de forma cooperativa junto con el resto de compañeros.

Aprender a dialogar y comunicarse entre sí fomenta las relaciones interpersonales. Por ejemplo, desde la asignatura de expresión corporal, se fomenta el diálogo (especialmente el de tipo corporal), ayudando a los alumnos a mejorar su imagen personal y a integrarse en el grupo, conectando el trabajo individual y colectivo. También con la finalidad de mejorar la capacidad dialógica de los estudiantes nos venimos valiendo de actividades de carácter cognitivo-linguísticas que les ayuden a: describir, definir, resumir, explicar, justificar, argumentar, demostrar sobre sus 
experiencias corporales de clase. Así por ejemplo, los estudiantes deberán valerse de diferentes tipos de lenguaje corporal, escrito o verbal para expresar lo realizado en la práctica; debiendo ser capaces de redactar resúmenes, parafrasear algunas ideas principales de los textos y elaborar esquemas y mapas conceptuales de los temas más relevantes.

Como otra parte de ese proceso comunicativo, los alumnos deben aprender a escuchar, a ponerse en lugar de los demás y, con ello, a cuestionar sus ideas y las de los demás, desde un respeto mutuo. Por ello, el alumnado debe aprender a: buscar información, observar, inferir, interpretar, explicar y debatir sobre problemáticas sociales vinculadas con la actividad corporal.

Para llevar a efecto esas capacidades, algunas de ellas de nivel superior, señalaremos algunas estrategias docentes:

- Invitar a los que menos participan a que repitan o resuman con sus propias palabras lo que se haya venido expresando en ese momento.

- Enseñar a distinguir entre el utilizar el nosotros cuando se venga realizando el trabajo en grupo o el yo pienso cuando se parte de experiencias y opiniones personales.

- Cuidar que prime el respeto entre los participantes.

- Mantener un contacto ocular y de escucha activa ante las opiniones de los estudiantes. No monopolizar el uso de la palabra.

- Esperar a mostrar su grado de acuerdo o desacuerdo, dejando que se genere un tiempo suficiente de debate para recoger diversas opiniones, siendo los estudiantes los que lleguen a emitir sus propias conclusiones.

- Ofrecer dilemas o preguntas abiertas que puedan ayudar a dicha discusión.

- Respetar los turnos de las palabras, favoreciendo a los que menos participan. Evitar que se formen subgrupos de opinión o que se centralice/monopolice entre pocos participantes.

- Controlar a nivel de tiempo las intervenciones personales y el conjunto del debate con objeto de poder atender a todas las cuestiones previstas. 
- Nombrar un secretario que tome nota de todo lo que se exponga (ya sea en la pizarra o en un documento que pueda llegar a todos los participantes) (Slavin, 1989; 1990; Fraile, 1997; López, 2005).

\subsection{Características del trabajo cooperativo}

- Los grupos no deben ser numerosos ya que se debe dar prioridad a los procesos de interacción.

- Se debe preparar a los estudiantes para actuar en grupos cooperativos

- Desarrollar habilidades básicas de interacción grupal (saber escuchar y respetar las opiniones ajenas, intentar los consensos, etc.).

- Resolver conflictos.

- Planificar y controlar el tiempo de las tareas.

- Motivar a los alumnos para llevar a cabo dicha tarea.

- Trabajar en grupos heterogéneos.

- Utilizar materiales que faciliten el trabajo grupal (Fraile, 2009b).

\subsection{Algunos obstáculos a superar a través del trabajo cooperativo:}

- El conformismo y la reducción de juicios críticos derivados de no ser excluidos al expresar ideas opuestas a la opinión generalizada del grupo.

- El control y la manipulación de los recursos por parte de unos pocos miembros del grupo.

- La reducción del esfuerzo individual.

- Aspectos relacionados negativamente con la toma de decisiones grupal como: la inhibición de algunos miembros del grupo, la dispersión de responsabilidades, la polarización de las decisiones, etc. (Fraile, 2009b).

Una vez expuestas las características de la evaluación formativa y del trabajo cooperativo se pasa a presentar una experiencia desarrollada con estudiantes de Educación Física, durante su formación inicial en la Facultad de Educación y Trabajo 
Social de la Universidad de Valladolid, España, y en la asignatura obligatoria de Expresión Corporal que se imparte en el $3^{\circ}$ curso de la titulación de Educación Primaria.

\section{Una experiencia de evaluación formativa a partir del aprendizaje cooperativo}

La experiencia que se recoge en este trabajo, parte de revisar el programa de la asignatura de Expresión Corporal a través del cual se ayuda, a estos estudiantes de Educación Física, a lograr un mejor conocimiento del cuerpo a la vez que se desarrollan unas determinadas capacidades interpersonales e intrapersonales como son: la empatía y la sensibilidad, la capacidad de expresar y canalizar sus afectos, la actividad cooperativa, el disfrute corporal desde de la actividad corporal, la necesidad de valorar la importancia del cuerpo desde la igualdad, las diferencias y la diversidad. Por ello, este conjunto de contenidos aparecen recogidos entre los aspectos a evaluar en el sistema de coevaluación formativa que es objeto de revisión en este trabajo que presentamos. A continuación, se recogen las diferentes fases y estrategias por las que pasan los estudiantes en su proceso formativo:

a) Considerar los conocimientos previos de los estudiantes. En el desarrollo del programa se parte de una primera tarea que supone que los estudiantes aprendan a reconocer y revisar sus experiencias previas y vivencias corporales desarrollada en las etapas previas a la Universidad. Para ello, se parte de considerar que la construcción de conocimientos se produce cuando lo que se aprende no sólo se debe a la nueva información presentada, sino también a los conocimientos previos de los aprendices (Pozo, 1994).

Con objeto de que los estudiantes adquieran, desde esta formación, una cultura académica y de aprendizaje seguimos un proceso de reconstrucción, que parte de sus conocimientos iniciales relacionados con sus concepciones, creencias, inquietudes, propósitos y actitudes. Ese conocimiento previo va unido a un proceso abierto de negociación sobre las finalidades, los contenidos y las estrategias docentes a seguir para conseguir dichos aprendizajes, buscando con ello conectar sus necesidades e intereses con las finalidades del propio programa de las asignatura.

Con ello, desde las primeras sesiones, los conocimientos básicos que los estudiantes han adquirido en su formación previa son revisados y utilizados con objeto conectar con los aprendizajes educativos a conseguir. Se les ayuda a cuestionar y 
problematizar esos conocimientos previos: provocando y facilitando la reconstrucción de un nuevo conocimiento, en este caso referido a la Educación Física y su relación con el mundo del deporte y la actividad física.

Para que los estudiantes puedan construir representaciones de su propio saber, deben aprender a interpretar sus experiencias de vida, desde sus conocimientos básicos. Por tanto, los resultados de su aprendizaje no sólo dependen del programa, sino de los conocimientos previos, de sus concepciones y motivaciones, entre otros aspectos, ya que el aprendizaje primero se adquiere y luego mejora cuando la nueva información se relaciona de forma significativa con lo que ya conocían.

Describir vivencias escolares, aportadas por los miembros del grupo, relacionar el tema de estudio con otros contenidos disciplinares. Recoger preguntas relativas al tema de estudio para el debate son tareas claves para que el estudiante sea consciente de sus vivencias personales vinculadas con la actividad corporal. Por ejemplo: cómo ese alumno en las etapas escolares se ha relacionado con el espacio, con el ritmo corporal, con su capacidad de crear gestos y movimientos, cómo hace consciente sus creencias sobre el cuerpo, cómo es capaz de revisar sus emociones, cómo se comunica con su cuerpo, etc. Por tanto, se trata de potenciar aquellas competencias expresivo-corporales que incluyen tanto habilidades de comunicación e indagación, como "know how" aplicadas al ejercicio de la profesión concreta de maestros de educación física (saber hacer).

b) Favorecer la construcción de aprendizajes significativos. Aprender de forma significativa representa que el estudiante pueda llegar a construir un conocimiento propio y personal (Ausubel, 1976). Por ello, una vez que los estudiantes han podido revisar sus creencias y conocimientos previos sobre los temas objeto de estudio, se les pide que los definan con su propio lenguaje, empleando ejemplos extraídos de sus vivencias prácticas, hasta obtener una mejor comprensión. Al enlazar los nuevos mensajes con sus conocimientos previos, acercamos la actividad docente a sus intereses.

Este proceso resulta más relevante cuando los estudiantes han pasado ya por el Practicum en el curso anterior, y con ello pueden exponer al resto de compañeros sus vivencias personales como docentes en prácticas. Eso les permite estar más disponibles para analizar los problemas surgidos en sus clases. Llegando a relacionar ese conocimiento teórico con las actividades desarrolladas por ellos en el aula, así como 
reflexionar sobre las estrategias empleadas en dichas prácticas. Por tanto, como parte de este proceso formativo, los estudiantes aprenden a: señalar el conocimiento declarativo (qué es lo que han aprendido), recoger en un mapa conceptual los conceptos básicos del tema, y hacer más consciente el cómo se ha llegado a aprender revisando cómo han ido construyendo ese aprendizaje.

\section{c) Estimular el aprendizaje autónomo de aprender a aprender a partir del trabajo}

grupal. El desarrollo de la autonomía como competencia personal se relaciona con el hecho de aprender, cuando quien aprende tiene la oportunidad de ejercer algún tipo de control procedimental, cognitivo o de proceso sobre el desarrollo del propio aprendizaje (Araujo y Sastre, 2008).

El trabajo grupal/colaborativo promueve la autonomía de los estudiantes, ya que ese trabajo colectivo parte de la construcción e intercambio de los aprendizajes individuales. Esto exige el dominio de diversas técnicas de estudio y estrategias cognitivas de aprender a aprender, dirigidas a conseguir una mayor comprensión de los contenidos, revisando qué hacen y por qué lo hacen. Este tipo de aprendizaje invita a los estudiantes a compartir los conocimientos, desde el contraste de opiniones y desde visiones divergentes. Para ello, hacemos uso del debate como estrategia que mejora la competencia de escuchar y comunicar, procurando crear un clima de respeto mutuo. Para Benito y Cruz (2005), el núcleo del aprendizaje cooperativo consiste en que los alumnos trabajen juntos para completar una tarea donde se preocupan tanto de su aprendizaje como el de sus compañeros (importancia de las relaciones interpersonales y de la autoestima).

Por tanto, con objeto de que los estudiantes puedan adquirir una mayor autonomía en el proceso de enseñanza-aprendizaje se utilizan procesos de negociación. Desde donde se establecen, de forma conjunta, las pautas y procedimientos para el diseño, la puesta en práctica y evaluación de las asignaturas. También se le hace intervenir en la selección de los materiales didácticos y la forma de aprenderlos, como parte de un proceso emancipador. Se les deja un tiempo para organizarse en grupos cooperativos desde sus intereses de aprendices y, por último, se le pide que se pongan de acuerdo en fijar los criterios para valorar los informes.

d) Promover el diálogo y el trabajo colaborativo donde se mejore el nivel de interacción y comunicación entre profesor y estudiantes. Desde un diálogo igualitario 
entre profesor y los estudiantes, siguiendo a Flecha (1998), se trata de potenciar entre los estudiantes un conocimiento compartido desde sus aportaciones y argumentos; en vez de valorar la posición de poder de quienes habitualmente lo realizan (el profesorado universitario). Desde un diálogo igualitario aprendemos ambos (profesor y estudiantes), cada uno desde sus interpretaciones y de acuerdo a unos intereses pactados previamente. Por tanto, el conocimiento compartido se va adquiriendo y construyendo tras el debate entre todos.

El aula representa el espacio favorecedor de este proceso abierto de diálogo y comunicación entre todos (profesor-estudiantes); en donde se comparte y revisan los conocimientos, creencias, vivencias, intereses, preocupaciones y deseos de todos; desde una mirada reflexiva y crítica de las diferentes visiones e interpretaciones de nuestras realidades educativas (ya que cada uno solemos interpretar de forma diferente nuestra propia realidad, siendo importante escuchar y analizar todas).

El profesor, como facilitador, es el responsable de crear las condiciones de comunicación e intercambio para que los estudiantes puedan presentar, sin miedo a equivocarse, sus pensamientos y creencias de manera crítica. Desde un lenguaje comprensible con el que avanzar en el conocimiento y en la acción. Para evitar que algunos acaparen el uso de la palabra, se les debe recordar la necesidad de respetar el turno de palabra, ayudándoles a superar el miedo a los silencios que son habituales en dichos debates, animando a participar a los que menos lo hacen, a exponer sus opiniones con su propio lenguaje, a analizar e interpretar el contenido de esos intercambios, etc.

Los estudiantes deben perder el miedo a compartir sus pensamientos y a responder a las preguntas que surgen en los debates. Ya que, más tarde ese conocimiento, elaborado de forma individual y grupal, será compartido con el resto de compañeros en la puesta en común final de clase.

Por tanto, para hacer efectivo ese diálogo entre iguales, hacemos uso de actividades y materiales de apoyo que permitan al profesor y a los estudiantes a interactuar. Y con ello, enriquecer ese proceso de aprendizaje. Para ello, los grupos de trabajo deben ser heterogéneos, atendiendo a la diversidad y a un proceso autónomo.

d) Ayudarles adquirir un pensamiento reflexivo y crítico sobre su práctica. El incremento de los canales de información en la sociedad del conocimiento nos exige no sólo 
saber elegir y seleccionar dichas fuentes, sino adquirir esas competencias de pensamiento reflexivo y crítico que ayude a los estudiantes a revisar su práctica. Con la finalidad de mejorar esa capacidad reflexiva y crítica, se proponen en clase una serie de actividades de carácter cognitivo-linguísticas que les ayuden a: describir, definir, resumir, explicar, justificar, argumentar, demostrar sobre sus experiencias corporales de clase. A partir de los diferentes tipos de lenguaje corporal, escrito o verbal deben ser capaces de redactar resúmenes, parafrasear algunas de las ideas principales, elaborar esquemas y mapas conceptuales. Así como organizar esa información y presentarla en clase.

Como parte de ese proceso reflexivo comunicativo, los estudiantes deben aprender a ponerse en lugar de los demás y, con ello, a cuestionar sus ideas o las de los otros, desde un respeto mutuo. A modo de ejemplo, Fernández Balboa (1999) nos sugiere algunos temas de discusión vinculados con la ética, con los valores y con la moralidad en el deporte.

Una vez presentados los procesos didácticos por los que se desarrolla la actividad docente, se presentan los indicadores de los que nos valemos para evaluar los aprendizajes de los estudiantes que se reflejan en sus informes grupales.

\subsection{Los criterios de evaluación de los informes grupales}

La principal de las tareas que realizan los estudiantes durante el curso es la elaboración de informes, unos de carácter semanal y otros final, siguiendo un proceso narrativo basado en desarrollar su competencia cooperativa y reflexiva. El contenido de los informes parte de los conocimientos previos del tema de estudio; para ello, se les comienza preguntando sobre los conocimientos básicos tratados en cursos anteriores, así como los recuerdos de las vivencias corporales relativas a cada uno de los temas que recoge la asignatura de Expresión Corporal. Dentro de este apartado se tendrán en cuenta que los estudiantes: a) Describen vivencias, aportadas por los miembros del grupo, relacionadas con el tema en una de las etapas escolares (primaria, secundaria) (extraescolares) o durante el Practicum en el centro escolar, b) Relacionan el tema de estudio con otros contenidos disciplinares presentando ejemplos ajustados a dicha temática y c) Elaboran preguntas que puedan ser debatidas en grupo relativas al tema de estudio. 
A continuación se recogen los conocimientos adquiridos del tema asignado, así como las estrategias para adquirirlos (cómo han sido aprendidos), destacando los cambios que se han producido, en cuanto a aprendizajes, desde los conocimientos previos, hasta el final del proceso. En este caso, las actividades realizadas durante el curso han sido: leer los artículos y extraer la información más relevante, debatir y compartir información con los compañeros, recoger las explicaciones del profesor, realizar los resúmenes de los textos, incorporar sugerencias y opiniones de otros compañeros, etc. En esta apartado se tiene en cuenta para su evaluación que los estudiantes: a) Elaboran una introducción que justifique la importancia del tema para su implementación en las clases de educación física en la etapa de educación primaria; b) Recogen definiciones relevantes del tema de forma sintética, indicando las fuentes bibliográficas de donde se extraen; c) Presentan teorías y fases del tema (desde los documentos escritos); d) Diseñan un sistema de evaluación relativo al tema de estudio: qué y cómo evaluar los contenidos del tema (presentarlo en cuadro) y d) Exponen las ventajas y obstáculos para el desarrollo del tema en la práctica escolar.

Otro de los componentes del informe son los esquemas o mapas conceptuales para sintetizar los principales contenidos del tema. En este caso, aparecen en dicho informe no sólo los contenidos aportados por el profesor en clase, sino los elaborados en los grupos cooperativos. Los criterios para su evaluación son: a) Los conceptos claves deben atender a los conocimientos adquiridos y al glosario de los principales conceptos del tema, b) debe establecerse una relación coherente y subordinada entre los conceptos claves y el resto de elementos; c) el mapa debe organizarse desde lo genérico a lo específico y cuidar que los diferentes elementos del mapa conceptual tengan algún tipo de relación, y d) se considera el número de aportaciones y la claridad de la presentación.

Después, el siguiente apartado incorpora aplicaciones didácticas del tema, con la intención de que posteriormente puedan llevarse a la práctica durante su periodo de Practicum con los escolares de Primaria. Para su diseño se consideran los elementos de un programa didáctico (objetivos, contenidos, metodología, competencias, recursos y sistema de evaluación). Los criterios para evaluar serán los siguientes: a) que esos diferentes elementos del programa estén claramente conectados; b) que atiendan las necesidades de los escolares relativas al tema de estudio; c) que la metodología fomente el carácter expresivo del alumnado y d) que la evaluación tenga un carácter formativo. 
Una vez finalizados los informes de cada tema, han de ser revisados y evaluados por el profesor y los estudiantes que asumen el papel de docentes. Con todos ellos se redactará uno global, desde un trabajo cooperativo de todo el grupo. Después se introducirá en la plataforma con objeto de ser revisado por el conjunto de la clase, pudiendo incluir aportaciones de todos los compañeros.

Una vez corregidos los informes finales y en las últimas semanas del curso, cada grupo presentará su Power Point, siendo coevaluados por el resto de compañeros a partir de criterios negociados con los estudiantes, relativos a:

- La presentación y nivel de redacción de los documentos presentados (ausencia de faltas ortográficas, uso de un lenguaje comprensible, utilización de ejemplos aclaratorios).

- Fundamentación teórica del tema y su posible transferencia a otras materias, nivel de razonamiento de los aspectos debatidos en clase, visión crítica ante las opiniones del profesor y resto de compañeros.

- Los cuadros, esquemas, mapas conceptuales en donde se sintetizan los contenidos presentados en clase (se valora las aportaciones personales a partir de lo elaborado en clase).

- Las aplicaciones didácticas sobre esos contenidos teóricos desarrollados en clase (sabe conectar la teoría con la práctica, propuestas para el Practicum, relaciones entre los diferente elementos del programa).

- La formulación de preguntas a partir de los textos (hipótesis de trabajo a partir de los textos leídos, relaciones entre diferentes teorías, cuestiones a ser observadas durante el Practicum).

- Un glosario terminológico donde se valora la capacidad de seleccionar información relevante y adecuarla desde un lenguaje significativo, sin perder el sentido y significado de los conceptos.

\subsection{Valoración del cumplimiento de los compromisos éticos}

Por último, también debemos evaluar cómo los estudiantes son capaces de adoptar un compromiso ético-moral vinculado a su desarrollo profesional (perspectiva crítica). 
Desde una educación democrática, nos debe preocupar que en nuestras clases se tengan presentes un conjunto de valores morales como son: el respeto, la tolerancia, la convivencia, la igualdad y la solidaridad, entre otros. Que se desarrollan a partir de las actitudes que exigen de una reflexión ética. Estos principios se deben hacer efectivos en la organización del aula y en las relaciones con los estudiantes, haciendo respetar las normas que rigen el comportamiento de la comunidad educativa.

Las relaciones democráticas con los estudiantes se reflejarán en la manera: de comunicarnos, de organizar el trabajo de clase, de atender, de escuchar y de respetar todo lo que sucede en el conjunto de la clase. Como parte de ese trato y respeto hacia la dignidad de los estudiantes. Es preciso llegar a romper el circuito de incapacidadfracaso-desinterés, ofreciendo situaciones atractivas, relevantes y significativas para su aprendizaje, desde un proceso de éxito.

Para atender la dimensión emocional de los estudiantes, se deberá respetar su sensibilidad y su deseo de saber, desde un compromiso moral; ya que la educación de los afectos debe ir conectada al aprendizaje de conocimientos. Para Rogers (1972), los educadores debemos ser capaces de comprender los significados y sentimientos del alumnado, siendo sensibles hacia sus actitudes. Esa educación de los valores morales se deberá ver reflejada en las normas de convivencia establecidas por los componentes de cada uno de los grupos. Por ejemplo: cómo se favorece en cada grupo las discusiones del grupo, cómo se respetan los principios de justicia y moralidad; cómo se obtiene una mejor resolución de los conflictos cuando surgen entre los miembros del grupo; cómo se produce la toma de decisiones desde el compromiso de buscar un buen funcionamiento del grupo.

Se trata de posibilitar en las clases una actuación autónoma y responsable de los estudiantes, animándoles a revisar lo que hacen y por qué lo hacen, tomando conciencia del proceso que siguen durante el aprendizaje grupal. Por ejemplo, se les trata de aportar protagonismo en el proceso de enseñanza-aprendizaje, invitándoles a compartir el diseño y revisión de los materiales curriculares, a revisar y modificar los criterios de evaluación, a asumir funciones de docentes con el resto de compañeros de clase, todo ello como parte de ese proceso emancipador. 
Entre algunos aspectos que serán objeto de evaluación están los que determinan la capacidad que tienen esos estudiantes de:

- ¿Reflexionar sobre su práctica siendo capaces de aplicar los resultados a la acción?

- ¿Desarrollar un tipo de relaciones democráticas de respeto, colaboración y solidaridad en el grupo?

- ¿Reconocer y dar solución a los problemas socio-morales que surgen en el grupo y en la clase?

- ¿Valorar la necesidad de desarrollar sus competencias profesionales?

- ¿Ser capaces de justificar sus decisiones relativas a la autoevaluación?

- ¿Adoptar un papel crítico ante la falta de conocimientos?

- ¿De comprometerse en participar en actividades fuera del horario académico que implique resolver situaciones o problemáticas de carácter social?

- ¿Identificar las cuestiones éticas que surgen en el proceso de enseñanzaaprendizaje?.

Para concluir, y como síntesis de las anteriores actividades de evaluación formativa, se les pide a los estudiantes que se sometan a un proceso de autoevaluación su actividad de aprendizaje. Las razones que justifican la presencia de la autoevaluación parten de la necesidad que tiene cada alumno de desarrollar su autonomía y responsabilidad como parte de su proceso de aprendizaje para ser docente. Con ello se favorece el desarrollo de valores educativos como son: la autonomía de aprendizaje, la honradez, la dignidad, el análisis crítico y la formación de personas responsables (López Pastor, 1999; Fraile, 2004).

Por tanto, cada estudiante debe redactar un breve documento en donde narra, entre otros aspectos, cómo se ha desarrollado, para él, dicho proceso de aprendizaje (qué competencias consideran que ha adquirido, cuáles son los conocimientos que considera más útiles, qué cambios haría en el programa para próximos cursos, cómo valora el aprendizaje cooperativo desarrollado en clase, qué opinión le merece el sistema de interacción profesor-estudiantes). Este uso de la autoevaluación se justifica al estar 
ligado al grado de confianza del profesor hacia el alumnado, lo que no exime de contrastar sus relatos con el cumplimiento de los criterios diseñados y negociados conjuntamente con ellos.

De cara a que el alumnado pueda autoevaluarse, cada uno de ellos ha podido tomar nota de qué forma evolucionan sus ideas recogidas en sus informes personales: cómo han ido superando las dificultades de aprendizaje, cómo han modificado sus estrategias para aprender, etc. No obstante, este proceso de autoevaluación tiene una relación muy directa con los compromisos que inicialmente cada uno de los estudiantes se ha propuesto conseguir, y la calificación final vendrá al comprobar el cumplimiento de esos compromisos.

A continuación, se recogen algunos criterios para ayudar a los estudiante a cómo valorar sus aprendizajes:

\section{Valoración de los aspectos cognitivos:}

Cada estudiante valorará la evolución de su aprendizaje desde el conocimiento previo hasta los adquiridos al finalizar cada uno de los temas, revisando aquellas pruebas que se han realizado con objeto de conocer el grado de conocimientos teóricos logrados durante el curso.

Los informes constituyen el principal medio para co-evaluar cada semana y al final del curso la capacidad del alumnado para hacer uso de diferentes competencias: buscar información, analizarla, sintetizarla, elaborar aplicaciones didácticas, valorar el trabajo de otros compañeros.

Los estudiantes tendrán un conocimiento de la valoración que hacen de su aprendizaje el profesor y sus compañeros (coevaluación), tanto cuando actúan como docentes, como después de que hayan revisado sus informes. Igualmente, en el trabajo grupal se asignará una calificación global para que luego se reparta, de forma consensuada, según los méritos de cada uno del grupo. También puede haber una atribución de la nota por igual entre todos.

Valoración de los aspectos procedimentales:

Se establecerán una serie de criterios para que cada estudiante valore su cuaderno de campo y los informes que realizan otros compañeros (coevaluación), considerando de forma negociada los siguientes criterios de valoración:

- Aspectos relativos a una buena presentación.

- Claridad en la redacción y ausencia de faltas ortográficas.

- Recoge las ideas principales debatidas en clase.

- Aporta nuevas ideas y argumentos después de leer los informes personales.

- Cuestiona y completa las ideas debatidas durante la clase.

- Expresa su visión particular del tema (revisión emocional).

- Construye nuevos mapas, cuadros o esquemas en donde se sintetiza la información más relevante de cada uno de los temas tratados.

- Busca diferentes aplicaciones didácticas a las ideas recogidas anteriormente.

- Genera nuevas preguntas que permita dar continuidad al ciclo de conocimientos. 
Valoración de los aspectos actitudinales:

Cada estudiante valorará su nivel de asistencia a lo largo del curso, teniendo como referencia la asistencia obligada al $80 \%$ total de horas de clase.

Valorará su nivel ético y de respeto hacia el resto de compañeros considerando que asiste a clase con objeto de colaborar con los grupos de aprendizaje, su autoevaluación se ajusta a los criterios y pautas definidas y consensuadas por el grupo (aunque en este caso el profesor supervisará dicha evaluación tras negociar y llegar a acuerdos con cada estudiante sobre la calificación final).

Valorará su participación en los debates y discusiones de pequeño y gran grupo.

Valorará su disposición a tomar iniciativas dentro del grupo, con objeto de avanzar en los debates. Así como las ayudas que realiza al resto de compañeros.

Todas las medidas que se pongan en marcha para incentivar el trabajo del alumno deben tener un reflejo en la calificación final de la asignatura. El problema con que nos encontramos, siempre que debemos poner una nota al trabajo del alumno, es ser justos con ese trabajo. Por ello apostamos por un procedimiento en el que los estudiantes se impliquen personalmente, a través de su autoevaluación, desde su autonomía y responsabilidad del aprendizaje, considerando que este proceso será un referente para su posterior etapa como profesores.

En diferentes momentos del curso cada estudiante revisará la consecución de sus compromisos, así como la valoración de cada uno ellos, justificando con las actividades realizadas la asignación de una calificación final. Las actividades que ha venido realizando con el objeto de cumplir con todos sus compromisos les lleva a valorar su actuación con un: Sobresaliente. Notable. Aprobado o Suspenso. Una vez que cada uno de ellos ha redactado su informe final de evaluación, deberá ratificarlo con el profesor para luego presentar en una asamblea con el resto de compañeros, debiendo justificar dicha petición. En el caso de que un alumno solicite la matrícula de honor esto será defendido ante el resto de compañeros los cuales, después de un breve debate, someterán a votación.

Por último, destacar que esta experiencia favorece una participación más democrática y responsable entre el alumnado, debiendo saber actuar ante la dificultad que representa esta forma de evaluar. No obstante, también la evaluación tradicional genera grandes problemas entre el profesorado ya que en muchas ocasiones no se utilizan los criterios o los instrumentos más adecuados para obtener una calificación justa.

Por tanto, adoptamos este criterio de autoevaluación justificado en que representa poder primar el tratamiento digno y de respeto hacia el alumno, desde una 
visión crítica, dejando que cada uno vaya adquiriendo con ello un compromiso responsable con su trabajo y sus resultados. Para concluir, se recoge el comentario realizado por uno de los estudiantes del curso: "si queremos que nuestros futuros alumnos sean capaces de autoevaluarse de una manera justa y responsable, debemos conocer dicho proceso desde la práctica para después educar en la justicia y en la igualdad de oportunidades, que les ayude a tomar decisiones basadas en esos principios".

\section{Consideraciones finales y conclusiones}

Entre los propósitos educativos que han estado presentes en nuestras aulas destacamos aquellos que han ayudado a los estudiantes, como futuros docentes, a actuar desde prácticas más democrática, basadas en un pensamiento crítico, donde se colabora uno con los otros y se trabaja en equipo, desde el respeto hacia las opiniones ajenas y del compromiso por ayudar a los compañeros más necesitados.

Gracias a los procesos de comunicación que hemos desarrollado en las aulas, los estudiantes se expresen de forma más abierta superando los miedos a compartir sus experiencias. En los debates en clase se han incrementado las interacciones entre el profesor y el alumnado, donde estos se han habituado a sincerarse y compartir sus problemáticas libremente. La comunicación entre los estudiantes ha favorecido el respeto, permitiéndonos observar en cada alumno cómo ha evolucionado su capacidad de aprender, sus creencias, sus formas de razonar, sus emociones y hábitos de trabajo.

Por último, hemos apreciado cómo a los estudiantes les preocupa cómo adquirir un nuevo modelo de transmisión del conocimiento que implique cambio sobre: cómo organizar los tiempos y el uso de los espacios en clase con sus alumnos de primaria, cómo atender mejor a los diferentes ritmos de aprendizaje, cómo buscar nuevas estrategias metodológicas para mejorar su aprendizaje cooperativo en el aula, cómo llegar a establecer un sistema de evaluación formativa y democrática que ayude a sus futuros estudiantes adquirir un pensamiento crítico.

Las ventajas más valoradas del sistema de coevaluación por los estudiantes son:

- Está centrado en el proceso, dando importancia al trabajo diario. 
- El trabajo se plantea en equipo de forma colaborativa.

- El estudiante realiza un aprendizaje activo.

- Hay interrelación entre la teoría y la práctica.

- Hay retroalimentación, posibilidad de corregir errores en documentos y actividades.

\section{Los inconvenientes más destacados en el sistema de coevaluación por los} estudiantes son:

- Exige continuidad.

- Exige una asistencia a clase obligatoria y activa.

- Tiene una dinámica de trabajo poco conocida.

- Exige un gran esfuerzo.

- Hay que compreder ese sistema de evaluación previamente.

\section{Referencias bibliográficas}

Álvarez Méndez, J.M. (1999). Diversidad y evaluación en educación. En Rubio y Rayón (coord.). Repensar la enseñanza desde la diversidad. Sevilla: Publicaciones MCEP.

Araujo, U.F. y Sastre, G. (2008). El aprendizaje basado en problemas. Barcelona: Gedisa.

Ausubel, D. (1976). Psicología educativa. México: Trillas.

Bain, K. (2006). Lo que hacen los mejores profesores universitarios. Valencia. PUV.

Barberá, E. (1999). Evaluación de la enseñanza, evaluación del aprendizaje. Barcelona: Edebé.

Benito, A. y Cruz, A. (2005). Nuevas claves para la docencia universitaria. Madrid. Narcea.

Bordás, M.I. y Cabrera, F.A. (2001). Estrategias de evaluación de los aprendizajes centrados en el proceso. Revista Española de Pedagogía, 218, 25-48. 
Boud, D. \& Falchikov, N. (ed.) (2007). Rethinking Assessment in Higher Education. Learning for the long term. Oxon, Routledge.

Brew, A. (2003). La autoevaluación y la evaluación por compañeros. En S. Brown y A. Glasner. (Ed.). Evaluar en la universidad. Problemas y nuevos enfoques. (179189). Madrid: Narcea.

Brown, S. (2003). Estrategias institucionales en evaluación. En Brown, S y Glasner, A. (Edit.): Evaluar en la Universidad. Problemas y nuevos enfoques. Madrid: Narcea.

Brown, S.; Race, P.; Rust, C. (1995). Using and experiencingassessment. En Knight, P. (Ed.): Assessment for Learning in Higher Education. Birmingham: Kogan Page/Staff and Educational. Development Association.

Brown, S., y Glasner, A. (Eds.) (2007). Evaluar en la Universidad. Problemas y nuevos enfoques. Madrid: Narcea.

Cano García, E. (2005). El portafolios del profesorado universitario. Un instrumento para la evaluación y para el desarrollo profesional. Barcelona: Octaedro.

Chomsky, N. (2001). La (Des) Educación. Barcelona: Crítica.

Dochy F., Segers, M., \& Sluijsmans, D. (1999). The Use of Self-, Peer and Coassessment in Higher Education: a review. Studies in Higher Education, 24, 3, 331-350.

Falchikov, N. \& Goldfinch, J. (2000). Student peer assessment in higher education: a meta-analysis comparing peer and teacher marks. Review of Educational Research, 70, 3, 287-322.

Falchikov, N. (2001). Learning together: peer tutoring in higher education. London: Routledge Falmer.

Falchikov, N. (2005). Improving Assessment Through Student Invlvement. Practical solutions for aiding learning in higher and further education. Oxon: Routledge.

Fernández Balboa, J.M. (1999). Pedagogía crítica y Educación Física en escuela secundaria. Revista Conceptos de Educación 6, 15-33. 
Fernández Balboa, J.M. (2005). La auto-evaluación como práctica promotora de la democracia y la dignidad. En A. Sicilia y J.M Fernández Balboa. La otra cara de la enseñanza. La Educación Física desde una perspectiva crítica. Barcelona: Inde.

Fernández-Balboa, J.M. (2006). Dignity and democracy in the college classroom: The practice of self-evaluation. In Rebecca A. Goldstein. Useful Theory: Making Critial Eduation Practical. New York. Peter Lang Publishing,

Flecha, R. (1998). Compartiendo palabras. Barcelona: Paidós.

Fraile Aranda, A. (1997). El trabajo cooperativo como propuesta de formación en Educación Física. Actas Congreso ICE de Zaragoza.

Fraile Aranda, A. (2004). Un cambio democrático en las aulas universitarias: Unas experiencia en la formación del profesorado de Educación Física. Contextos educativos: Revista de Educación, 6-7, 213-234.

Fraile Aranda, A. (2006). Cambios en el aula universitaria ante los nuevos retos europeos. Tándem, 20, 57-72.

Fraile Aranda, A. (2009a). El Espacio Europeo de Enseñanza Superior: Un controvertido camino para la formación y el cambio educativo del profesorado universitario. Revista Universidades. 40, 3-17. Universidad de México.

Fraile Aranda, A. (2009b). Un sistema común de aprendizaje cooperativo y evaluación formativa para dos asignaturas. En V. López Pastor. Evaluación formativa y compartida en Educación Superior. (183-190). Madrid: Narcea.

Freire, P. (1970). Pedagogía del oprimido. Madrid: Siglo XXI.

Habermas, J. (1989). El discurso filosófico de la modernidad. Barcelona: Taurus.

Ibarra, S; Rodríguez, G y Gómez, M.A. (2012). La evaluación entre iguales: beneficios y estrategias para su práctica en la universidad. Revista de Educación, 359, 206231.

Johnson, D; Johnson, R. (1975). Learning togheter and alone. Englewood Cliffs: Prentice Hall. 
Johnson, D.; Johnson, R., y Holubec, E. (1999). El aprendizaje cooperativo en el aula. Barcelona: Paidós.

Johnston, M. (1997). El significado de la colaboración más allá de las diferencias culturales. Revista Kikiriki, 46. MCEP.

López Noguera, F. (2005). Metodología participativa en la enseñanza universitaria. Madrid: Narcea

López Pastor, V.M. (coord.) (2006). La Evaluación en Educación Física. Revisión de los modelos tradicionales y planteamiento de una alternativa: la evaluación formativa y compartida. Buenos Aires: Miño y Dávila.

López Pastor, V.M. (coord.) (2009). La Evaluación Formativa y Compartida en la docencia universitaria. Madrid: Narcea.

Marina, J.A. (1995). Etica para náufragos. Barcelona: Anagrama

Macdowell, L. y Sambell, K. (2003). La experiencia en la evaluación innovadora. En S. Brown, y A. Glasner (Edit.) (2003). Evaluar en la Universidad. Problemas y nuevos enfoques. Madrid: Narcea.

Monereo, C. (Coord.). (2009). Pisa como excusa. Barcelona: Graó.

Morin, E. (2000). La mente bien ordenada. Barcelona: Seix Barral.

Porlan, R. y Martín, J. (1991). El diario del profesor. Sevilla: Diada.

Prins, F.J., Sluijsmans, M.A., Kirschner, P.A. \& Strijbos, J-W. (2005). Formative peer assessment in a CSCL evironment: a case study. Assessment \& Evaluation in Higher Education, 30, 4, 417-444.

Rogers, C. (1972). El proceso de convertirse en persona. Barcelona: Paidós.

Slavin, R. (1989). Cooperative learning theory. Research and Practice. Englewood Cliffs. N.J: Prentice-Hall.

Slavin, R. (1990) Research on cooperative learning: consensus and controversy. Educar Leadership. 47, 4, 52-55.

Stenhouse, L.(1987). La investigación como base de la enseñanza. Madrid. Morata. 
Tejedor, F. J. (2002). Actitudes y conductas habituales de los profesores de enseñanza obligatoria en relación con la evaluación de los alumnos. Revista de Educación, 328, 325-354.

Velázquez, C; Fraile, A y López Pastor, V. (2014). Aprendizaje Cooperativo en Educación Física. Movimento. 20, 1, 239-259. 